\title{
A case study on early detection, prediction and prevention of heart disease in a multispecialty hospital by applying six sigma methodologies
}

\author{
V. Nirmala ${ }^{*}$, K.K. Suresh ${ }^{2}$ \\ ${ }^{1}$ Women Scientist - A, Department of Science and Technology, Govt. of India (DST-WOS-A), Department of Statistics, \\ Bharathiar University, Coimbatore-641046, Tamil Nadu, India. \\ ${ }^{2}$ Department of Statistics, Bharathiar University, Coimbatore, Tamil Nadu, India \\ "Corresponding Author: vnirmalakanna@yahoo.com, Tel.: 9787099896.
}

Available online at: www.isroset.org

Accepted 18/Aug/2018, Online 30/Aug/2018

\begin{abstract}
Survey report on predicting positive Coronary Artery Disease from symptoms and evolving a diagnostic package for the 128 sliced computed tomography for a multispecialty hospital by using six sigma methodologies. Materials and methods Dynamic 128-slice Computed Tomography Scan was performed in patients admitted to the radiology department among October 2013 to January 2014. This paper deals with the data based decision making process by implementing Zero defect quality, Six Sigma methodologies. The presentation makes an attempt to study the "Existing process of numerous data generation" towards zero defect quality and make the Computed Tomography Scan diagnostic intelligent technology aware among the common man in bringing awareness on "Early Detection, Prediction and Prevention". To continuously upgrade the technology for an early diagnosis and to render excellent quality treatment to the patients.
\end{abstract}

Key words - Six Sigma, Quality, DMAIC, Breakthrough improvement, mini tab 17 software.

\section{INTRODUCTION}

Motorola engineering scientist William Smith, known as the father of Six Sigma [4], developed the concept in the 1980s. For many years, he and other pioneering engineers and scientists worked on this or similar concepts to reduce variation, improve quality. The methodology of Six Sigma uses the statistical theory and thus assumes that every process factor can be characterized by a statistical distribution curve $[8-10,16]$.

This work is a case study of Six Sigma [1], using the DMAIC improvement methodology, can be applied in a hospital environment. It is based on a project done by 81-person in multispecialty hospital in Coimbatore city in India. Continuously striving hard for "Early detection of Heart Disease" (CAD- coronary artery disease) particularly for the increase in the proportion of $60 \%$ of world's heart patients will be contributed by India (By 2010 WHO).

Adapting technological innovations [3] of "Intelligent systems" installing Computed Tomography Scan (CT scan ) according to low and steady rate heart rates with lowest radiation (Dose 1-3mSv) (Adaptive cardio sequence) and high and steady rate heart rates (Adaptive ECG pulsing )
(Dose 7-8mSv) Bringing out the right mix of machine and men to achieve brake through improvement [2].

The rest of this paper is organized as follows. In the next Section II the scope and design objective are described. Section III contains the methodology, measures and approach. Section IV contains statement of the problem, analysis, results and discussion is expressed by figures and tables to observe the significant symptoms of the disease. Section $\mathrm{V}$ presents the conclusion of the study in future directions.

\section{SCOPE AND DESIGN OBJECTIVE}

Scope of the study

The presentation makes an attempt to study the "existing process of numerous data generation" towards zero defect quality [5] and to make the CT scan diagnostic intelligent technology aware among the common man in bringing awareness on "Early Detection, Prediction and Prevention".

\section{Goal}

To continuously upgrade the Technology for an early diagnosis and to render excellent quality treatment to the patients.

\section{Sigma}


By definition sigma (s), a Greek letter, is the statistical quality measurement of standard deviation from the mean. Six Sigma [6] describes how a process performs quantitatively. In other words, it measures the variation of performance.

\section{Six Sigma}

Six sigma is a tool used to convert management problem into a statistical problem and to find a statistical solution then convert it to a Management solution [12].

\section{Design for six sigma steps: (DMAIC)}

- Define the project goals and customer (internal and external) requirements.

- Measure and determine customer needs and specifications (LSL and USL); benchmark competitors and similar industries.

- Analyze the process or product options to meet customer needs.

- Improve (detailed) the process or product to meet customer needs.

- Control the design performance and ability to meet customer needs $[13,14]$.

\section{DEFINE PHASE}

\section{The strategic issue for the Hospital}

Even at the highest rate of Rs. 12,000 per test, requires 6000 Patients per year to meet the Capital cost of investment alone (assuming Rs. 7.2 Crore's as the investment cost). Here comes the patient affordability cost and the availability volume. The Vital factor is the "Accessibility" of patients and building the communication in public, making patients as promoters through excellent, predictable and reliable service levels in order to prevent CAD.

\section{Focus Area}

CT-SCAN with reference to Cardiac applications.

\section{Objective}

Work out a high risk prone group in the society and to prepare a package for the diagnosis.

\section{"HIGH QUALITY, LOW COST - HIGH VOLUME PROGRAM"}

\section{The Economy Aspect}

The Quality is not just giving the output, but making a "Process Plan" using the best use of "Voluminous Information " in discovering the pattern and evolving (not Patient focused treatment alone, but assessing the clinical trends for accelerating the Quality and the outcome) a "Patient - Focused Scheme" - according to the risk prone groups.

\section{III . METHODOLOGY, MEASURES AND APPROACH}

In assessing the clinical trends for accelerating the Quality and the outcome, a "Patient - Focused Scheme" - according to the risk prone groups is considered.

6000 patients to be tested per year, just to meet the Capital cost of Rs. 7.2 Crore's without the operational cost (i.e. Salary, Maintenance, Consumables and Stationeries), it means 20 patients per day or 500 patients in a month of 25 days to be diagnosed for CAD. Currently the average arrival is only 80 to 110 patients per month. How India is to upgrade technology despite high probability of CAD.

\section{MEASURE PHASE}

\section{Data source}

The hospital records Existing hard copies and case-data of individual patients for the months of October 2013 to January 2014.

\section{Base line information}

Quality of arriving patients Excluding Age, Sex, Urban/Rural the patient may arrive with previous history of defect events such as Hypertension, Smoking, Chest Pain, H/o MI, H/o PTCA , CABG and Diabetics. As such the defect in arrival rate (i.e. a patient will have at least one defect Event) is $83 \%$. It means symptomatic people only had come for diagnosis mostly, but the results also show non-symptomatic people recording positive coronary artery disease (CAD).

\section{Six sigma is a Benchmark:}

The "Process" needs to be defined. It may not be possible, that a company attains "Benchmark" in all process. But it should be possible, to establish, to develop, to identified and compare with "someone" as a role model to grow and achieve as a continuous improvement. Six-sigma [6] demands performance improvements through numerical rating.

\section{Glossary of symbols:}

DPU - Defect per Unit

DPO - Defect per Opportunity

$\mathrm{P}$ - Probability

PPM - Parts per million opportunity

$\mathrm{Z}-\mathrm{Z}$ value (Normal curve value) 
A focus on classical and six sigma approach:

\begin{tabular}{|c|c|c|}
\hline \multirow[t]{2}{*}{ Issue } & \multicolumn{2}{|c|}{ Focus } \\
\hline & Classical & Six sigma \\
\hline 1. Management & Cost \& time & $\begin{array}{l}\text { Quality \& } \\
\text { time }\end{array}$ \\
\hline 2. Goal setting & $\begin{array}{l}\text { Realistic } \\
\text { perception }\end{array}$ & $\begin{array}{c}\text { Reach-out \& } \\
\text { stretch }\end{array}$ \\
\hline 3. Direction & Seat-of-pant & $\begin{array}{c}\text { Benchmarking } \\
\text { \&metrics }\end{array}$ \\
\hline $\begin{array}{l}\text { 4. Chain of } \\
\text { command }\end{array}$ & Hierarchy & $\begin{array}{c}\text { Empowered } \\
\text { team }\end{array}$ \\
\hline 5. Focus & Product & Process \\
\hline 6. Approach & Symptomatic & Problematic \\
\hline 7. Problems & Fixing & Preventing \\
\hline 8. Reasoning & $\begin{array}{l}\text { Experience } \\
\text { based }\end{array}$ & $\begin{array}{c}\text { Statistically } \\
\text { based }\end{array}$ \\
\hline $\begin{array}{l}\text { 9. Problem } \\
\text { solving }\end{array}$ & Expert based & $\begin{array}{c}\text { System } \\
\text { based }\end{array}$ \\
\hline $\begin{array}{l}\text { 10.Analytical } \\
\text { perspective }\end{array}$ & $\begin{array}{c}\text { Point } \\
\text { estimate }\end{array}$ & Variability \\
\hline 11. Design & Performance & Productivity \\
\hline $\begin{array}{l}\text { 12. Variable } \\
\text { search }\end{array}$ & $\begin{array}{l}\text { One-factor-at-a- } \\
\text { time }\end{array}$ & $\begin{array}{c}\text { Design of } \\
\text { experiments }\end{array}$ \\
\hline 13. Tolerance & Worst case & $\begin{array}{l}\text { Root-sum-of- } \\
\text { squares }\end{array}$ \\
\hline $\begin{array}{c}14 . \\
\text { Manufacturing }\end{array}$ & Trial and error & Robust design \\
\hline $\begin{array}{l}\text { 15. Process } \\
\text { monitoring }\end{array}$ & $\begin{array}{l}\text { Specification } \\
\text { limits }\end{array}$ & SQC charts \\
\hline $\begin{array}{l}\text { 16. Process } \\
\text { adjustment }\end{array}$ & fixed & Beta correction \\
\hline
\end{tabular}

\section{STATEMENT OF THE PROBLEM, ANALYSIS, RESULTS}

\section{AND DISCUSSION}

Problem Statement No. 1

Quality at data retrieval reducing the incidence of "Non availability" of patient's prior history information's in the hospital. Among 480 patients only 81 patients could be retrieved (among 10 opportunities of information age, Sex, Urban/Rural, Hypertension, Smoking, Chest Pain, H/o MI, H/o PTCA, CABG, Diabetics) for full history details which need to be achieved $100 \%$. The other symptoms like chest pain, heart rate, were not captured for some of the patients.
Arrival Quality of Patients Improving the "Defect free" prior history of Patients from $83 \%$ of patients by Social Awareness program. The existing arrival rate of defect event per opportunities of history information is the effect of age, gender, and rural/urban place as a promotional factors (or) symptoms. It is only to be considered as promotional factors. (Please note now the arrivals are mostly symptomatic) [10]. Vispute and Singh [16] developed an economic acceptance sampling plan for variables under the method of second order auto correlation. In these problem "Positive" cases of CAD Among the patients arrival, the incidence of positive cases of CAD influenced by history information would drive home the prevention of severity of attacks-angina and the counseling required to increase patient's reference for longevity of life.

\section{Coded Index}

Coded index data were calculated by using Minitab 17 software, in ANALYSE PHASE
Total defects =
137 (for the 7 defects alone)

DPU $\quad=\quad 1.6914($ Unit $=$ Patient $)$

$\mathrm{DPO} \quad=\quad 0.241622575$

$\begin{array}{lll}\mathrm{P} & = & 0.214647467\end{array}$

$\begin{array}{lll}\mathrm{PPM} & =214647\end{array}$

$\begin{array}{lll}\mathrm{Z} & =0.7904\end{array}$

SIGMA LEVE $\quad \mathrm{L}=2.2904$

No. Of Patients $\quad=81$

Positive Reported $=46$

Positive Percentage $=56.79012$

The six sigma [5] statistical method in order to achieve the zero defect in any process.

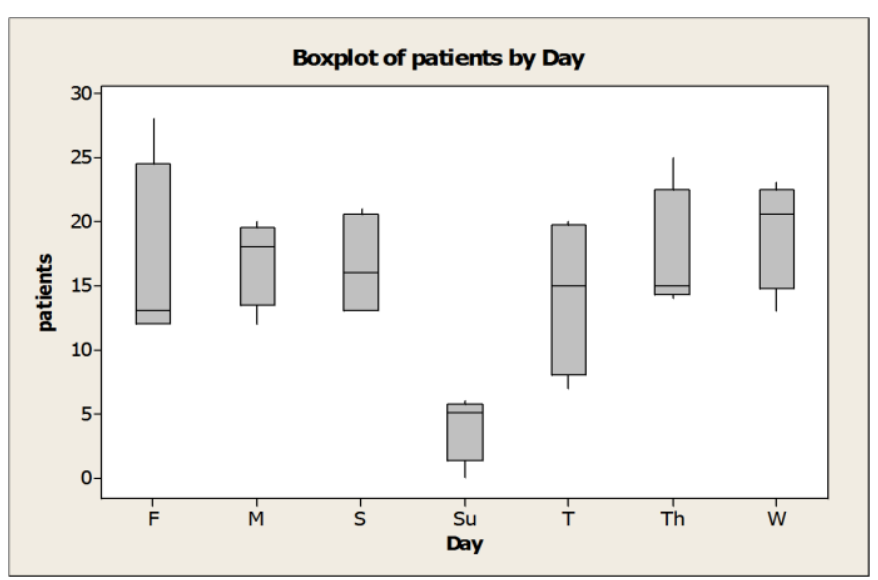

BOX PLOT: (mini tab 17)

To view the central tendency \& highlight the variability in the data [7]

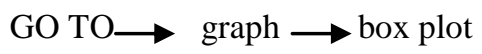

Problem Statement No. 2 
Individual95\% Class Intervalsfor Mean Based on Pooled standard deviation

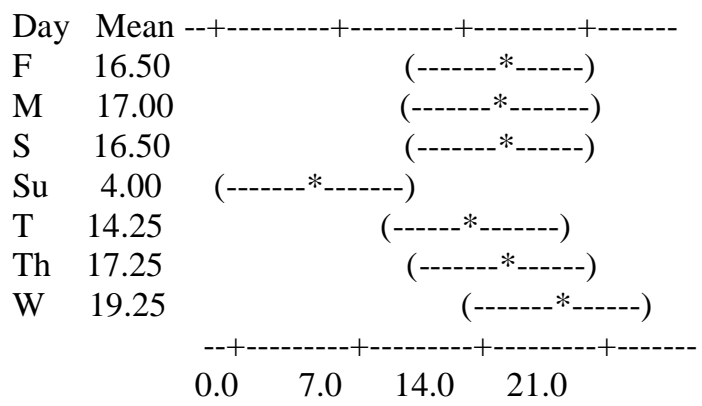

Individual 95\% CIs For Mean Based on Pooled St. Dev
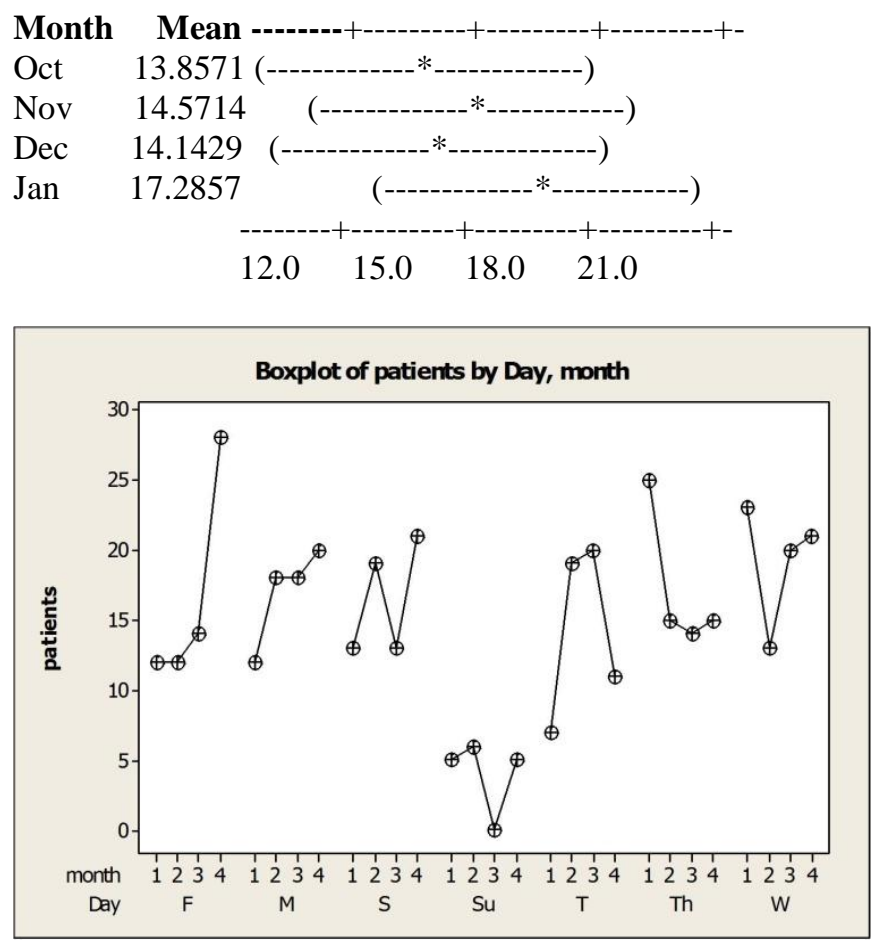

Link Function: Logistic

Response Information

\begin{tabular}{|l|c|l|}
\hline Variable & Value & Count \\
\hline Coded: Results & 1 & 46 (Event) \\
\hline & 0 & 35 \\
\hline Total & & 81 \\
\hline
\end{tabular}

\section{LOGISTIC REGRESSION}

\begin{tabular}{|c|c|c|c|c|c|c|c|}
\hline \multicolumn{6}{|c|}{ Logistic Regression } & \multicolumn{2}{|c|}{ 95\% C.I. } \\
\hline Predictor & Co.ef. & SE Co.ef. & $\begin{array}{c}\text { Z- } \\
\text { Val } \\
\text { ue }\end{array}$ & $\begin{array}{c}\text { P- } \\
\text { Val } \\
\text { ue }\end{array}$ & $\begin{array}{l}\text { Odds } \\
\text { Ratio }\end{array}$ & $\begin{array}{c}\text { Low } \\
\text { er }\end{array}$ & $\begin{array}{l}\text { Up } \\
\text { per }\end{array}$ \\
\hline Constant & -12.77 & 3024082 & $\begin{array}{c}- \\
3.94\end{array}$ & 0.0 & - & - & - \\
\hline Age & 0.219 & 0.0550224 & 4.00 & 0.00 & 1.25 & 1.12 & $\begin{array}{c}1.3 \\
9\end{array}$ \\
\hline $\operatorname{Sex}(M)$ & 1.09 & 0.914123 & 1.19 & 0.23 & 2.98 & 0.50 & $\begin{array}{l}17 . \\
85\end{array}$ \\
\hline $\begin{array}{l}\text { Urban/R } \\
\text { ural (U) }\end{array}$ & 0.53 & 0.760697 & 0.70 & 0.47 & 1.70 & 0.38 & $\begin{array}{c}7.5 \\
5\end{array}$ \\
\hline $\begin{array}{c}\text { Hyperten } \\
\operatorname{sion}(\mathbf{Y})\end{array}$ & 0.03 & 0.710855 & 0.04 & 0.97 & 1.03 & 0.26 & $\begin{array}{c}4.1 \\
6\end{array}$ \\
\hline $\begin{array}{l}\text { Smoking } \\
\text { (Y) }\end{array}$ & -1.55 & 1.14820 & $\overline{1.35}$ & 0.18 & 0.21 & 0.02 & $\begin{array}{c}2.0 \\
2\end{array}$ \\
\hline $\begin{array}{c}\text { Chest } \\
\text { Pain }(\mathbf{Y})\end{array}$ & 0.35 & 0.793377 & 0.45 & 0.66 & 1.43 & 0.30 & $\begin{array}{c}6.7 \\
5\end{array}$ \\
\hline $\begin{array}{c}\text { H/o MI } \\
\text { (Y) }\end{array}$ & 22.18 & 9144.96 & 0.00 & 0.99 & $\begin{array}{c}4.282 \\
07 E+ \\
09\end{array}$ & 0.00 & $*$ \\
\hline $\begin{array}{c}\text { H/o } \\
\text { PTCA } \\
(\mathbf{Y})\end{array}$ & $\begin{array}{c}2.1536 \\
9\end{array}$ & 1.44589 & 1.49 & $\begin{array}{c}0.13 \\
6\end{array}$ & 8.62 & 0.51 & $\begin{array}{l}146 \\
.59\end{array}$ \\
\hline $\begin{array}{c}\text { CABG } \\
(\mathbf{Y})\end{array}$ & $\begin{array}{c}19.455 \\
6\end{array}$ & 7798.33 & 0.00 & $\begin{array}{c}0.99 \\
8\end{array}$ & $\begin{array}{c}2.814 \\
87 E+ \\
08\end{array}$ & 0.00 & $*$ \\
\hline $\begin{array}{c}\text { Diabetic } \\
\text { (P) }\end{array}$ & $\begin{array}{c}0.8256 \\
13\end{array}$ & 0.756888 & 1.09 & $\begin{array}{c}0.27 \\
5\end{array}$ & 2.28 & 0.52 & $\begin{array}{l}10 . \\
07\end{array}$ \\
\hline
\end{tabular}

Log-Likelihood $=\mathbf{- 2 7 . 6 5 7}$

Test that all slopes are zero: $G=55.478, D F=10, \underline{P}$-Value $=\mathbf{0 . 0 0 0}$

The data analysis showed that there is at least one factor which establishes as significant symptom for reporting positive CAD.This is analyzed by factor wise from the $\mathrm{P}$ value.

Measures of Association:

(Between the Response Variable and Predicted Probabilities)

\begin{tabular}{|c|c|c|c|c|}
\hline Pairs & $\begin{array}{c}\text { Num } \\
\text { ber }\end{array}$ & $\begin{array}{c}\text { Perc } \\
\text { ent }\end{array}$ & $\begin{array}{c}\text { Summ } \\
\text { ary }\end{array}$ & $\begin{array}{c}\text { Measu } \\
\text { res }\end{array}$ \\
\hline $\begin{array}{c}\text { Concord } \\
\text { ant }\end{array}$ & 1484 & 92.2 & Somers' D & 0.84 \\
\hline $\begin{array}{c}\text { Discorda } \\
\text { nt }\end{array}$ & 124 & 7.7 & $\begin{array}{c}\text { Goodman } \\
\text {-Kruskal } \\
\text { Gamma }\end{array}$ & 0.85 \\
\hline Ties & 2 & 0.1 & $\begin{array}{c}\text { Kendall's } \\
\text { Tau-a }\end{array}$ & 0.42 \\
\hline Total & 1610 & 100.0 & & \\
\hline
\end{tabular}




\section{IMPROVE PHASE}
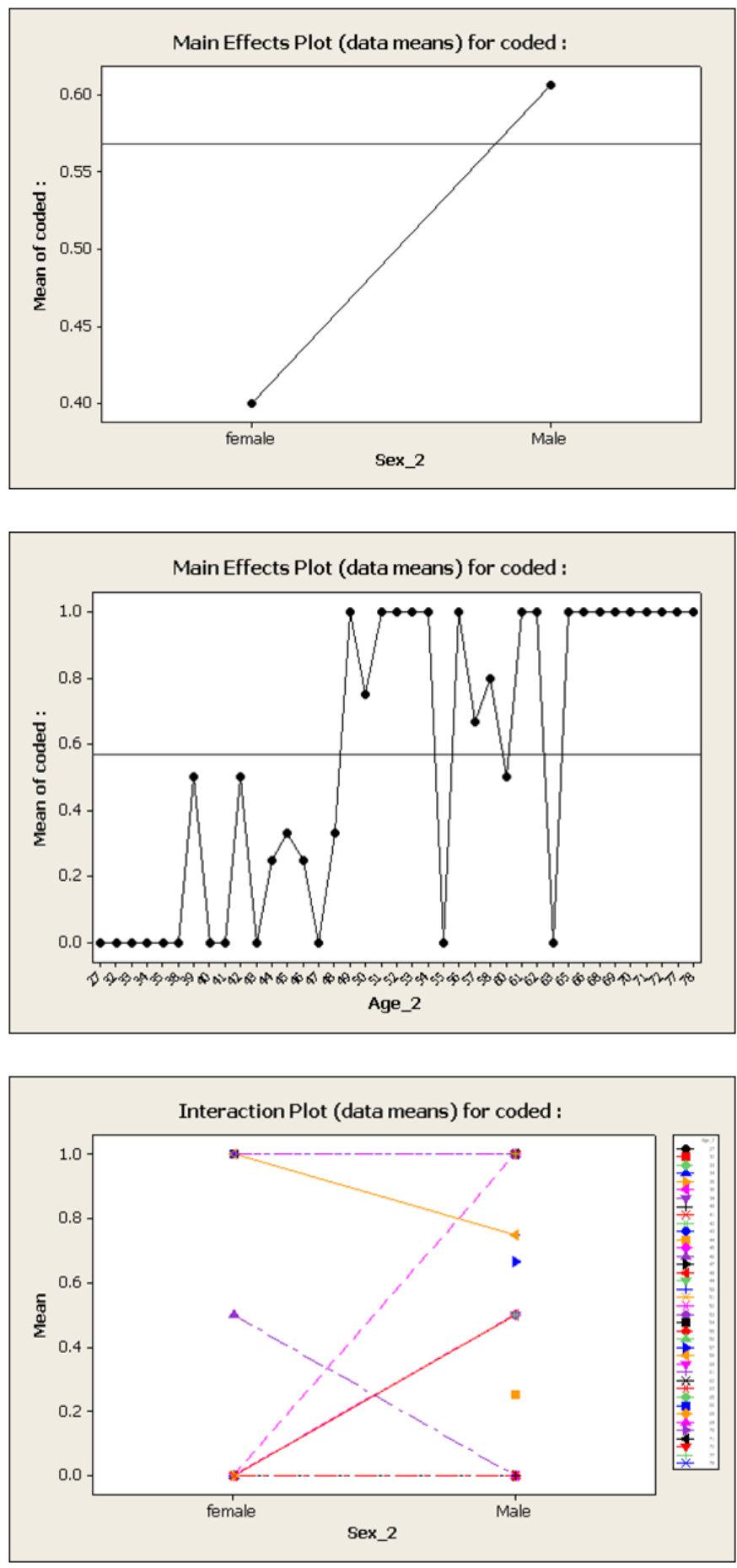

\section{CONTROL PHASE}

Age:

The age has severe effect of reporting positive CAD with almost $100 \%$ lower limits with the odds of 1.25 times of younger people below 40 years of age. This report is shown in the main effect.

\begin{tabular}{|c|c|c|c|c|c|c|c|c|c|c|c|c|}
\hline $\begin{array}{l}\text { S. } \\
\mathbf{N} \\
\text { o. }\end{array}$ & $\begin{array}{l}\mathbf{A} \\
\mathrm{g} \\
\mathbf{e}\end{array}$ & $\begin{array}{l}\mathbf{S} \\
\mathbf{e} \\
\mathbf{x}\end{array}$ & $\begin{array}{l}\text { Ur } \\
\text { ba } \\
\text { n/ } \\
\text { Ru } \\
\text { ral }\end{array}$ & $\begin{array}{l}\text { Hy } \\
\text { per } \\
\text { Ten } \\
\text { sion }\end{array}$ & $\begin{array}{l}\mathbf{S} \\
\mathbf{~ m} \\
\mathbf{o} \\
\mathbf{k i} \\
\mathbf{n} \\
\mathbf{g}\end{array}$ & $\begin{array}{l}\text { C } \\
\text { he } \\
\text { st } \\
\text { Pa } \\
\text { in }\end{array}$ & $\begin{array}{c}\text { H } \\
\text { /o } \\
\text { M } \\
\text { I }\end{array}$ & $\begin{array}{c}\text { H/ } \\
\mathbf{0} \\
\text { PT } \\
\text { C } \\
\text { A }\end{array}$ & $\begin{array}{l}\text { CA } \\
\text { BG }\end{array}$ & $\begin{array}{c}\text { Dia } \\
\text { beti } \\
\text { c } \\
\text { P/N }\end{array}$ & $\begin{array}{c}\text { Co } \\
\text { de } \\
\text { d } \\
\text { Re } \\
\text { sul } \\
t\end{array}$ & $\begin{array}{l}\text { No.d } \\
\text { ef/pa } \\
\text { t }\end{array}$ \\
\hline 1 & $\begin{array}{l}4 \\
9\end{array}$ & 1 & 1 & 1 & 0 & 0 & 0 & 0 & 0 & 0 & 1 & 1 \\
\hline 2 & $\begin{array}{l}4 \\
6\end{array}$ & 0 & 1 & 0 & 0 & 1 & 0 & 1 & 0 & 1 & 1 & 3 \\
\hline 3 & $\begin{array}{l}4 \\
6\end{array}$ & 0 & 1 & 0 & 0 & 0 & 0 & 0 & 0 & 0 & 0 & 0 \\
\hline 4 & $\begin{array}{l}4 \\
4\end{array}$ & 1 & 1 & 0 & 0 & 0 & 0 & 0 & 0 & 0 & 0 & 0 \\
\hline 5 & $\begin{array}{l}7 \\
8\end{array}$ & 0 & 0 & 1 & 0 & 1 & 0 & 0 & 0 & 1 & 1 & 3 \\
\hline 6 & $\begin{array}{l}6 \\
8\end{array}$ & 1 & 1 & 0 & 0 & 1 & 0 & 0 & 0 & 0 & 1 & 1 \\
\hline 7 & $\begin{array}{l}6 \\
0\end{array}$ & 1 & 1 & 0 & 0 & 1 & 0 & 0 & 1 & 0 & 1 & 2 \\
\hline 8 & $\begin{array}{l}4 \\
0\end{array}$ & 1 & 1 & 1 & 0 & 0 & 0 & 0 & 0 & 0 & 0 & 1 \\
\hline 9 & $\begin{array}{l}4 \\
5\end{array}$ & 1 & 0 & 0 & 0 & 1 & 0 & 0 & 0 & 0 & 1 & 1 \\
\hline $\begin{array}{l}1 \\
0\end{array}$ & $\begin{array}{l}6 \\
6 \\
\end{array}$ & 1 & 0 & 0 & 0 & 1 & 0 & 0 & 1 & 1 & 1 & 3 \\
\hline $\begin{array}{l}1 \\
1\end{array}$ & $\begin{array}{l}5 \\
6\end{array}$ & 1 & 0 & 1 & 1 & 0 & 1 & 0 & 0 & 0 & 1 & 3 \\
\hline $\begin{array}{l}1 \\
2\end{array}$ & $\begin{array}{l}5 \\
8\end{array}$ & 0 & 1 & 1 & 0 & 0 & 0 & 0 & 0 & 1 & 1 & 2 \\
\hline $\begin{array}{l}1 \\
3\end{array}$ & $\begin{array}{l}6 \\
2\end{array}$ & 1 & 1 & 0 & 0 & 1 & 0 & 0 & 0 & 0 & 1 & 1 \\
\hline $\begin{array}{l}1 \\
4 \\
\end{array}$ & $\begin{array}{l}6 \\
1 \\
\end{array}$ & 1 & 1 & 1 & 0 & 0 & 0 & 0 & 0 & 0 & 1 & 1 \\
\hline $\begin{array}{l}1 \\
5\end{array}$ & $\begin{array}{l}5 \\
0\end{array}$ & 1 & 1 & 0 & 1 & 0 & 0 & 0 & 0 & 1 & 1 & 2 \\
\hline $\begin{array}{l}1 \\
6 \\
\end{array}$ & $\begin{array}{l}5 \\
8 \\
\end{array}$ & 1 & 1 & 1 & 0 & 0 & 0 & 0 & 0 & 0 & 1 & 1 \\
\hline $\begin{array}{l}1 \\
7\end{array}$ & $\begin{array}{l}7 \\
0\end{array}$ & 1 & 1 & 1 & 0 & 1 & 0 & 0 & 1 & 1 & 1 & 4 \\
\hline $\begin{array}{l}1 \\
8\end{array}$ & $\begin{array}{l}4 \\
0\end{array}$ & 1 & 0 & 0 & 0 & 1 & 0 & 0 & 0 & 1 & 0 & 2 \\
\hline $\begin{array}{l}1 \\
9\end{array}$ & $\begin{array}{l}5 \\
0 \\
\end{array}$ & 1 & 0 & 1 & 1 & 1 & 1 & 0 & 0 & 0 & 1 & 4 \\
\hline $\begin{array}{l}2 \\
0\end{array}$ & $\begin{array}{l}3 \\
9 \\
\end{array}$ & 1 & 1 & 1 & 0 & 1 & 0 & 1 & 0 & 1 & 1 & 4 \\
\hline $\begin{array}{l}2 \\
1 \\
\end{array}$ & $\begin{array}{l}6 \\
8 \\
\end{array}$ & 1 & 1 & 1 & 0 & 1 & 0 & 0 & 0 & 1 & 1 & 3 \\
\hline $\begin{array}{l}2 \\
2\end{array}$ & $\begin{array}{l}6 \\
5 \\
\end{array}$ & 1 & 0 & 1 & 0 & 1 & 0 & 0 & 0 & 1 & 1 & 3 \\
\hline $\begin{array}{l}2 \\
3\end{array}$ & $\begin{array}{l}5 \\
0\end{array}$ & 1 & 0 & 1 & 0 & 0 & 0 & 0 & 0 & 1 & 1 & 2 \\
\hline $\begin{array}{l}2 \\
4\end{array}$ & $\begin{array}{l}5 \\
1\end{array}$ & 1 & 0 & 0 & 0 & 0 & 0 & 0 & 1 & 0 & 1 & 1 \\
\hline $\begin{array}{l}2 \\
5\end{array}$ & $\begin{array}{l}4 \\
3 \\
\end{array}$ & 1 & 0 & 1 & 0 & 1 & 0 & 0 & 0 & 0 & 0 & 2 \\
\hline $\begin{array}{l}2 \\
6\end{array}$ & $\begin{array}{l}77 \\
7\end{array}$ & 1 & 1 & 0 & 0 & 1 & 0 & 0 & 0 & 1 & 1 & 2 \\
\hline $\begin{array}{l}2 \\
7 \\
\end{array}$ & $\begin{array}{l}7 \\
1 \\
\end{array}$ & 1 & 0 & 1 & 1 & 0 & 1 & 0 & 1 & 1 & 1 & 5 \\
\hline $\begin{array}{l}2 \\
8\end{array}$ & $\begin{array}{l}4 \\
4 \\
\end{array}$ & 1 & 1 & 0 & 0 & 1 & 0 & 0 & 0 & 0 & 1 & 1 \\
\hline $\begin{array}{l}2 \\
9\end{array}$ & $\begin{array}{l}4 \\
4 \\
\end{array}$ & 1 & 0 & 1 & 0 & 0 & 0 & 0 & 0 & 1 & 0 & 2 \\
\hline 3 & 4 & 1 & 0 & 1 & 0 & 0 & 0 & 0 & 0 & 1 & 0 & 2 \\
\hline
\end{tabular}




\begin{tabular}{|c|c|c|c|c|c|c|c|c|c|c|c|c|}
\hline 0 & 6 & & & & & & & & & & & \\
\hline $\begin{array}{l}3 \\
1\end{array}$ & $\begin{array}{l}6 \\
1\end{array}$ & 0 & 0 & 1 & 0 & 1 & 0 & 0 & 0 & 0 & 1 & 2 \\
\hline $\begin{array}{l}3 \\
2\end{array}$ & $\begin{array}{l}5 \\
5\end{array}$ & 1 & 1 & 1 & 0 & 0 & 0 & 0 & 0 & 0 & 0 & 1 \\
\hline $\begin{array}{l}3 \\
3\end{array}$ & $\begin{array}{l}5 \\
2\end{array}$ & 1 & 1 & 1 & 0 & 1 & 0 & 0 & 0 & 0 & 1 & 2 \\
\hline $\begin{array}{l}3 \\
4\end{array}$ & $\begin{array}{l}5 \\
3\end{array}$ & 1 & 1 & 0 & 0 & 0 & 0 & 1 & 0 & 0 & 1 & 1 \\
\hline $\begin{array}{l}3 \\
5\end{array}$ & $\begin{array}{l}4 \\
1\end{array}$ & 1 & 0 & 1 & 0 & 1 & 0 & 0 & 0 & 1 & 0 & 3 \\
\hline $\begin{array}{l}3 \\
6\end{array}$ & $\begin{array}{l}6 \\
2\end{array}$ & 1 & 1 & 1 & 0 & 0 & 0 & 0 & 0 & 1 & 1 & 2 \\
\hline $\begin{array}{l}3 \\
7\end{array}$ & $\begin{array}{l}6 \\
2\end{array}$ & 1 & 0 & 1 & 0 & 1 & 0 & 0 & 0 & 1 & 1 & 3 \\
\hline $\begin{array}{l}3 \\
8\end{array}$ & $\begin{array}{l}5 \\
7\end{array}$ & 1 & 1 & 1 & 0 & 1 & 0 & 0 & 0 & 1 & 1 & 3 \\
\hline $\begin{array}{l}3 \\
9\end{array}$ & $\begin{array}{l}4 \\
5\end{array}$ & 0 & 0 & 0 & 0 & 0 & 0 & 0 & 0 & 1 & 0 & 1 \\
\hline $\begin{array}{l}4 \\
0\end{array}$ & $\begin{array}{l}7 \\
2\end{array}$ & 1 & 0 & 0 & 0 & 0 & 0 & 0 & 0 & 0 & 1 & 0 \\
\hline $\begin{array}{l}4 \\
1\end{array}$ & $\begin{array}{l}5 \\
6\end{array}$ & 1 & 1 & 1 & 0 & 0 & 0 & 0 & 0 & 0 & 1 & 1 \\
\hline $\begin{array}{l}4 \\
2\end{array}$ & $\begin{array}{l}3 \\
2\end{array}$ & 0 & 1 & 0 & 0 & 0 & 0 & 0 & 0 & 0 & 0 & 0 \\
\hline $\begin{array}{l}4 \\
3\end{array}$ & $\begin{array}{l}3 \\
5\end{array}$ & 0 & 0 & 1 & 0 & 1 & 0 & 0 & 0 & 0 & 0 & 2 \\
\hline $\begin{array}{l}4 \\
4\end{array}$ & $\begin{array}{l}5 \\
7\end{array}$ & 1 & 0 & 0 & 0 & 1 & 0 & 0 & 0 & 1 & 0 & 2 \\
\hline $\begin{array}{l}4 \\
5\end{array}$ & $\begin{array}{l}4 \\
2\end{array}$ & 1 & 0 & 0 & 0 & 0 & 0 & 0 & 0 & 0 & 0 & 0 \\
\hline $\begin{array}{l}4 \\
6\end{array}$ & $\begin{array}{l}4 \\
1\end{array}$ & 1 & 1 & 0 & 0 & 0 & 0 & 0 & 0 & 0 & 0 & 0 \\
\hline $\begin{array}{l}4 \\
7\end{array}$ & $\begin{array}{l}6 \\
8\end{array}$ & 1 & 1 & 0 & 1 & 1 & 1 & 0 & 1 & 0 & 1 & 4 \\
\hline $\begin{array}{l}4 \\
8\end{array}$ & $\begin{array}{l}5 \\
4\end{array}$ & 0 & 1 & 1 & 0 & 0 & 0 & 0 & 0 & 0 & 1 & 1 \\
\hline $\begin{array}{l}4 \\
9\end{array}$ & $\begin{array}{l}6 \\
3\end{array}$ & 1 & 1 & 0 & 1 & 0 & 0 & 0 & 0 & 0 & 0 & 1 \\
\hline $\begin{array}{l}5 \\
0\end{array}$ & $\begin{array}{l}3 \\
9\end{array}$ & 1 & 1 & 0 & 0 & 0 & 0 & 0 & 0 & 0 & 0 & 0 \\
\hline $\begin{array}{l}5 \\
1\end{array}$ & $\begin{array}{l}4 \\
1\end{array}$ & 1 & 0 & 0 & 0 & 0 & 0 & 0 & 0 & 0 & 0 & 0 \\
\hline $\begin{array}{l}5 \\
2\end{array}$ & $\begin{array}{l}6 \\
9\end{array}$ & 1 & 0 & 0 & 0 & 0 & 0 & 0 & 0 & 1 & 1 & 1 \\
\hline $\begin{array}{l}5 \\
3\end{array}$ & $\begin{array}{l}6 \\
0\end{array}$ & 0 & 0 & 1 & 0 & 1 & 0 & 0 & 0 & 0 & 0 & 2 \\
\hline $\begin{array}{l}5 \\
4\end{array}$ & $\begin{array}{l}3 \\
4\end{array}$ & 0 & 0 & 0 & 0 & 0 & 0 & 0 & 0 & 1 & 0 & 1 \\
\hline $\begin{array}{l}5 \\
5\end{array}$ & $\begin{array}{l}4 \\
7\end{array}$ & 1 & 0 & 1 & 1 & 0 & 0 & 0 & 0 & 1 & 0 & 3 \\
\hline $\begin{array}{l}5 \\
6\end{array}$ & $\begin{array}{l}7 \\
7\end{array}$ & 1 & 1 & 0 & 0 & 0 & 0 & 0 & 0 & 0 & 1 & 0 \\
\hline $\begin{array}{l}5 \\
7\end{array}$ & $\begin{array}{l}4 \\
7\end{array}$ & 0 & 1 & 0 & 0 & 1 & 0 & 0 & 0 & 1 & 0 & 2 \\
\hline $\begin{array}{l}5 \\
8\end{array}$ & $\begin{array}{l}3 \\
8\end{array}$ & 1 & 0 & 0 & 0 & 0 & 0 & 0 & 0 & 0 & 0 & 0 \\
\hline $\begin{array}{l}5 \\
9\end{array}$ & $\begin{array}{l}4 \\
8\end{array}$ & 1 & 0 & 0 & 1 & 1 & 1 & 0 & 0 & 0 & 1 & 3 \\
\hline $\begin{array}{l}6 \\
0\end{array}$ & $\begin{array}{l}4 \\
1\end{array}$ & 0 & 1 & 0 & 0 & 0 & 0 & 0 & 0 & 0 & 0 & 0 \\
\hline $\begin{array}{l}6 \\
1\end{array}$ & $\begin{array}{l}6 \\
8\end{array}$ & 1 & 1 & 1 & 0 & 1 & 0 & 0 & 0 & 0 & 1 & 2 \\
\hline $\begin{array}{l}6 \\
2\end{array}$ & $\begin{array}{l}4 \\
5\end{array}$ & 1 & 1 & 0 & 0 & 0 & 0 & 0 & 0 & 1 & 0 & 1 \\
\hline
\end{tabular}

\begin{tabular}{|c|c|c|c|c|c|c|c|c|c|c|c|c|}
\hline $\begin{array}{l}6 \\
3\end{array}$ & $\begin{array}{l}7 \\
2 \\
\end{array}$ & 1 & 0 & 1 & 1 & 0 & 0 & 0 & 0 & 0 & 1 & 2 \\
\hline $\begin{array}{l}6 \\
4 \\
\end{array}$ & $\begin{array}{l}5 \\
7\end{array}$ & 1 & 1 & 0 & 0 & 0 & 0 & 0 & 0 & 1 & 1 & 1 \\
\hline $\begin{array}{l}6 \\
5\end{array}$ & $\begin{array}{l}5 \\
8 \\
\end{array}$ & 1 & 0 & 0 & 1 & 0 & 0 & 0 & 0 & 0 & 1 & 1 \\
\hline $\begin{array}{l}6 \\
6\end{array}$ & $\begin{array}{l}4 \\
1\end{array}$ & 1 & 1 & 0 & 1 & 1 & 0 & 0 & 0 & 0 & 0 & 2 \\
\hline $\begin{array}{l}6 \\
7\end{array}$ & $\begin{array}{l}5 \\
1 \\
\end{array}$ & 1 & 0 & 0 & 0 & 0 & 0 & 0 & 0 & 1 & 1 & 1 \\
\hline $\begin{array}{l}6 \\
8\end{array}$ & $\begin{array}{l}4 \\
8\end{array}$ & 1 & 1 & 0 & 0 & 1 & 0 & 0 & 0 & 0 & 0 & 1 \\
\hline $\begin{array}{l}6 \\
9 \\
\end{array}$ & $\begin{array}{l}5 \\
2\end{array}$ & 1 & 0 & 1 & 0 & 0 & 0 & 0 & 0 & 1 & 1 & 2 \\
\hline $\begin{array}{l}7 \\
0\end{array}$ & $\begin{array}{l}2 \\
7\end{array}$ & 1 & 1 & 1 & 1 & 0 & 0 & 0 & 0 & 0 & 0 & 2 \\
\hline $\begin{array}{l}7 \\
1\end{array}$ & $\begin{array}{l}3 \\
8\end{array}$ & 1 & 1 & 0 & 0 & 1 & 0 & 0 & 0 & 0 & 0 & 1 \\
\hline $\begin{array}{l}7 \\
2\end{array}$ & $\begin{array}{l}4 \\
8\end{array}$ & 0 & 1 & 1 & 0 & 1 & 0 & 0 & 0 & 1 & 0 & 3 \\
\hline $\begin{array}{l}7 \\
3 \\
\end{array}$ & $\begin{array}{l}5 \\
2\end{array}$ & 0 & 1 & 0 & 0 & 1 & 0 & 0 & 0 & 1 & 1 & 2 \\
\hline $\begin{array}{l}7 \\
4\end{array}$ & $\begin{array}{l}5 \\
8\end{array}$ & 1 & 1 & 1 & 0 & 0 & 0 & 0 & 0 & 0 & 0 & 1 \\
\hline $\begin{array}{l}7 \\
5\end{array}$ & $\begin{array}{l}3 \\
3\end{array}$ & 1 & 0 & 0 & 1 & 1 & 0 & 0 & 0 & 0 & 0 & 2 \\
\hline $\begin{array}{l}7 \\
6\end{array}$ & $\begin{array}{l}4 \\
6\end{array}$ & 1 & 1 & 1 & 1 & 0 & 0 & 1 & 0 & 1 & 0 & 4 \\
\hline $\begin{array}{l}7 \\
7\end{array}$ & $\begin{array}{l}5 \\
8\end{array}$ & 1 & 1 & 0 & 0 & 0 & 0 & 0 & 0 & 0 & 1 & 0 \\
\hline $\begin{array}{l}7 \\
8\end{array}$ & $\begin{array}{l}6 \\
6\end{array}$ & 1 & 1 & 1 & 1 & 0 & 0 & 0 & 0 & 0 & 1 & 2 \\
\hline $\begin{array}{l}7 \\
9\end{array}$ & $\begin{array}{l}5 \\
0\end{array}$ & 1 & 1 & 0 & 0 & 0 & 0 & 0 & 0 & 0 & 0 & 0 \\
\hline $\begin{array}{l}8 \\
0\end{array}$ & $\begin{array}{l}4 \\
2\end{array}$ & 1 & 1 & 1 & 0 & 0 & 0 & 0 & 0 & 1 & 1 & 2 \\
\hline $\begin{array}{l}8 \\
1\end{array}$ & $\begin{array}{l}4 \\
4\end{array}$ & 1 & 1 & 1 & 0 & 0 & 0 & 0 & 0 & 1 & 0 & 2 \\
\hline
\end{tabular}

\section{Smoking / Nonsmoker:}

Note the smoker as a factor event and note the negative sign coefficient, it means nonsmoker has also odds of reporting positive CAD at $83 \%$ confidence level.

Sex:

Male has got three times more chance than the Female to be reported as positive $\mathrm{CAD}$

\begin{tabular}{|l|l|l|l|l|}
\hline Sex & Male & $\mathbf{1}$ & Female & $\mathbf{0}$ \\
\hline Urban/ Rural & Urban & $\mathbf{1}$ & Rural & $\mathbf{0}$ \\
\hline Hypertension & Positive & $\mathbf{1}$ & Negative & 0 \\
\hline Smoking & Positive & $\mathbf{1}$ & Negative & $\mathbf{0}$ \\
\hline Chest Pain & Positive & $\mathbf{1}$ & Negative & 0 \\
\hline H/o MI & Positive & $\mathbf{1}$ & Negative & 0 \\
\hline H/o PTCA & Positive & 1 & Negative & 0 \\
\hline H/o CABG & Positive & $\mathbf{1}$ & Negative & 0 \\
\hline Diabetics & Positive & $\mathbf{1}$ & Negative & 0 \\
\hline
\end{tabular}




\begin{tabular}{|c|l|}
\hline Sigma level & PPM level \\
\hline 2 & $3,08,537$ \\
3 & 66210 \\
4 & 6210 \\
5 & 233 \\
6 & 3.4 \\
\hline
\end{tabular}

PTCA:

It is obvious that PTCA patients have got 9 times more chance than the non PTCA patients for reporting positive CAD.

\section{CABG:}

It is also obvious that $\mathrm{CABG}$ patients have 3 times more chance than the non PTCA patients for reporting Positive CAD. The remaining factors such as urban/rural, hypertension, chest pain, H/O MI, Diabetics do not have significant effect.

\section{CONCLUSION}

The age has severe effect of reporting positive CAD with almost $100 \%$ lower limits with the odds of 1.25 times of younger people below 40 years of age. This report is shown in the main effect. Note the smoker as a factor event and note the negative sign coefficient, it means nonsmoker has also odds of reporting positive CAD at $83 \%$ confidence level. Male has got three times more chance than the Female to be reported as positive CAD. It is obvious that PTCA patients have got 9 times more chance than the non PTCA patients for reporting positive $\mathrm{CAD}$. It is also obvious that $\mathrm{CABG}$ patients have 3 times more chance than the non PTCA patients for reporting Positive CAD. The remaining factors such as urban/rural, hypertension, chest pain, H/O MI, Diabetics do not have significant effect.

\section{Acknowledgements}

The first author is thankful to Ministry of Science and Technology, Department of Science and Technology (DST), Govt. of India, New Delhi for providing Women Scientist - A and Principal Investigator of DST-WOS-A research project for financial support to carry out of this research work and grateful to Bharathiar University, for providing necessary facilities for doing research project. Prof. A. Rajagopal ISI Coimbatore, helped me for Six Sigma analysis. The second author is thankful to University Grants Commission, New Delhi for providing UGC - OTG for research program and DST-FIST, UGC - SAP programmes.

\section{REFERENCES}

[1]. Albright S. "Data Analysis and Decision Making with Microsoft Excel”, Duxbury Press, ISBN: 0534389325 pp. 5672, 2001 .

[2]. Breakthrough Management Group, "Six Sigma Black belt Training Handbook". Tamko Roofing Products, Total Quality Management Handbook pp. 32-61.

[3]. Chowdhary, S., "The power of Six Sigma: An Inspiring Tale of How Six Sigma Is Transforming the Way We Work", Dearborn Trade, ISBN: 0793144345. Pp. 64-79, 2001.

[4]. Delrue L, Blanckaert P, Mertens D, et al. “Assessment of tumor vascularization in pancreatic adenocarcinoma using 128-slice perfusion CT imaging". J Comput Assist Tomogr Vol. 35, PP. 434 - 438, 2011

[5]. Deming, W., "Out of the Crisis", MIT Press, ISBN: 0262541157, Vol. 1, pp. 3-16, 2000.

[6]. Forrest W. Breyfogle III, "Managing Six Sigma: A Practical Guide to Understanding, Assessing, and Implementing the Strategy That Yields Bottom-Line Succes, WileyInterscience", ISBN: 0471396737, Vol. 3, Issue 1, pp. 5-11, 2000.

[7]. M. Mounica A, Ananda Rao, P. Radhika Raju, "PABR Algorithm for Improving the Data archival performance of a HDFS", International Journal of Computer Science and Engineering Vol.6, Issue-7, pp.37-42, 2018

[8]. Nirmala.V and Suresh. K. K., "Construction and Selection of Continuous Sampling Plan of type (CSP-T) Indexed through Maximum Allowable Average Outgoing Quality". Journal of Statistics and Management System. Vol. 20, Issue 3: pp. 4414572017.

[9]. Nirmala.V and Suresh. K. K. "Designing of Bayesian Skip lot Sampling Plan - V with MDS (0, 2)", International Journal of Statistics and Analysis, Vol. 7, Issue 1, pp. 15-18, 2017.

[10]. Nirmala.V and Suresh. K. K., "Designing of MATLAB Program for Various Fuzzy Quality Regions in CSP-MLP-T3 Sampling plan", International Journal on Future Revolution in Computer Science and Communication Engineering, Vol. 3, Issue 7, pp. 065-068, 2017.

[11]. Nirmala.V and Suresh. K. K., "Designing of Continuous Sampling Plan of type (CSP-3) indexed through Maximum Allowable Average Outgoing Quality with Markov chain approach", International Journal of Statistics and Systems, Vol. 12, Issue 2, pp. 277-283, 2017.

[12]. Pande, P., et al, "The Six Sigma Way: How GE, Motorola, and Other Top Companies are Honing Their Performance", McGaw-Hill Trade, ISBN: 0071358064, Vol. 5, Issue 1, pp. 11-27, 2000.

[13]. Pyzdek T., "The Six Sigma Handbook, McGraw-Hill Trade", ISBN: 0071372334, Vol. 8, Issue 1, pp. 51-124, 2000.

[14]. P.A. Wange and S.M. Rajbhoj, "Energy Efficient Industrial Application Using IOT", International Journal of Computer Science and Engineering Vol.6, Issue-7, pp.115-118, 2018.

[15]. R. Radhakrishnan and P. K. Sivakumaran, "Prob Stat Forum Construction and selection of six sigma based Link sampling plans", Vol. 3,pp. 65-77, 2010,

[16]. S. Vispute, J.R. Singh, "Economic Effect on Variables Sampling Plans Under Second Order Auto-Correlation", 
International Journal of scientific research in Mathematical and Statistical Sciences, Vol. 1, Issue. 2, pp.1-15, 2014.

[17]. Suresh.K.K and Nirmala.V, "Designing of Continuous Sampling Plan (CSP-T) through Quality Decision Regions", International Journal of Pure and Applied Mathematics, Vol. 106, Issue 7, pp. 15 - 20, 2016.

\section{AUTHORS PROFILE}

Dr. V. Nirmala is a Women Scientist - A, Department of Science and Technology, Govt. of India (DST-WOS-A), Department of Statistics, Bharathiar University, Coimbatore641046, Tamil Nadu, India. Pursued M.Sc., M.Phil., P.G.D.C.A., and Ph.D., in Statistics from Bharathiar University, Coimbatore. She has done Six Sigma Green and Black Belt programme in Indian Statistical Institute (ISI).

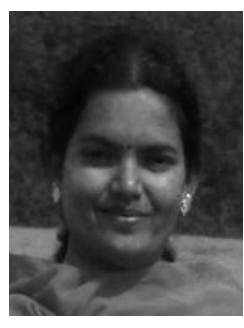

She is currently doing as Principal Investigator in a major Research Project in Department of Statistics, Bharathiar University, Coimbatore. She has published more than 20 research papers in reputed international journals, it's also available online. Her main research work focuses on Statistical Quality Control, Acceptance Sampling, Six sigma Methodologies, TQM.

Prof. Dr. K. K. Suresh is a Professor and Head, Department of Statistics, Bharathiar University, Coimbatore, Tamil Nadu, India. He has more than 35 years of Research and Teaching experience in the field of Statistics and has published more than 300 Research papers in national and international journals.

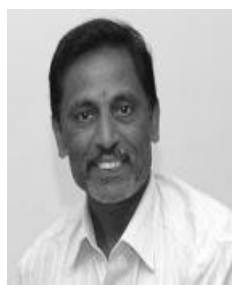

